\title{
Long term experience using the ADAPT technique for the treatment of acute ischemic stroke
}

\author{
Jan Vargas, ${ }^{1}$ Alejandro Spiotta, ${ }^{1}$ Kyle Fargen, ${ }^{1}$ Raymond Turner, ${ }^{1}$ Imran Chaudry, ${ }^{2}$ \\ Aquilla Turk ${ }^{2}$
}

'Department of Neurosurgery, Medical University of South Carolina, Charleston, South Carolina, USA 2Department of Radiology, Medical University of South Carolina, Charleston, South Carolina, USA

Correspondence to Dr J Vargas, Department of Neurosciences, Medical University of South Carolina, 96 Jonathan Lucas Street, CSB 301 Charleston, SC 29425, USA; Vargasma@musc.edu

Received 30 November 2015 Revised 24 March 2016 Accepted 28 March 2016 Published Online First 18 April 2016

\section{CrossMark}

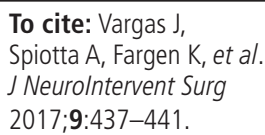

\section{ABSTRACT}

Introduction The direct aspiration first pass technique (ADAPT) has been introduced as a simple and fast method for achieving good angiographic and clinical outcomes using large bore aspiration catheters for the treatment of acute ischemic stroke (AIS). We present a single center's long term experience with ADAPT.

Methods Retrospective analysis of a database was gathered on patients undergoing stroke thrombectomy with ADAPT at a stroke center. Specific parameters captured included age, gender, National Institutes of Health Stroke Scale (NIHSS) score at presentation, time to presentation from last normal, and modified Rankin Scale (mRS) score at the 90 day follow-up. Radiological and angiographic imaging was reviewed to document the location of the vascular occlusion, Thrombolysis in Cerebral Infarction (TICI) flow postprocedure, and procedural complications.

Results 191 consecutive patients who suffered an AIS treated with ADAPT were reviewed; 91 were women, and mean age was 67 years. Patients presented with a mean NIHSS score of 15.4, and 71 patients received intravenous tissue plasminogen activator. The average time from onset to puncture was $7.8 \mathrm{~h}$. The average time for recanalization was $37.3 \mathrm{~min}$. TICI $2 \mathrm{~B}$ or better recanalization was achieved in 180 (94.2\%) patients. 98 $(54.1 \%)$ patients had an mRS of $0-2$ at 90 days. Direct aspiration alone was performed in 145 cases, and 43 cases required the additional use of a stent retriever. There was no significant difference in presenting NIHSS score, average time to presentation, average $\mathrm{mRS}$ at 90 days, or 90 day mortality between the two groups. Time to recanalization was 29.6 min for direct aspiration compared with 61.4 min in cases that required adjunct devices ( $p=0.00000201) .79(57.7 \%)$ patients who underwent direct aspiration only achieved a good outcome at 90 days (mRS $0-2$ ) compared with 19 (43.2\%) who underwent adjunct therapies $(p=0.12)$.

Conclusions ADAPT is an effective method to achieve good clinical and angiographic outcomes, and serves as a useful firstline method for revascularization.

\section{INTRODUCTION}

Acute ischemic stroke (AIS) remains a major burden on the healthcare system in the USA, with an estimated 134000 deaths annually. It is widely accepted that faster recanalization of occluded vessels and reperfusion of ischemic territories has been associated with improved outcomes following acute stroke. ${ }^{1}$ Recently, multiple randomized clinical trials were completed demonstrating improved outcomes in patients with ischemic stroke who undergo endovascular thrombectomy compared with intravenous thrombolysis alone. ${ }^{2-6}$ The success of these recent trials is attributable to using advanced imaging to select patients with large vessel occlusions (LVO), focusing on rapid progression from triage to endovascular therapy and using modern thrombectomy devices and techniques. ${ }^{7}$

The development of newer revascularization devices has yielded faster and more complete recanalizations and subsequent outcomes compared with older technologies. ${ }^{8} 9$ The direct aspiration first pass technique (ADAPT) was recently introduced as an efficient and cost effective thrombectomy method for achieving excellent angiographic and clinical outcomes using large bore aspiration catheters. ${ }^{10} 11$ This technique has now been used as the primary revascularization technique since 2012 at our institution. The purpose of the present study is to report our ongoing experience with ADAPT.

\section{METHODS}

\section{Database}

A retrospective analysis of a prospectively maintained database was performed to identify all patients undergoing thrombectomy for LVO with ADAPT at our institution using an institutional review board approved protocol. Patients undergoing thrombectomy between December 2012 and April 2015 were included. Specific parameters captured included age, gender, National Institutes of Health Stroke Scale (NIHSS) score at presentation, and time to presentation from last normal. Of significance, angiograms were blindly graded by an independent neurointerventionalist (KF) to assess pre- and post-revascularization using both the Thrombolysis in Cerebral Ischemia (TICI; with scores of $0,1,2 \mathrm{~A}, 2 \mathrm{~B}$, and 3$)^{12}$ flow post procedure and the modified 2C TICI grading scale (including scores of $0,1,2 \mathrm{~A}, 2 \mathrm{~B}, 2 \mathrm{C}$, and 3 ). ${ }^{13}$ The interventionalist was at a separate institution during the time period of the data acquisition, and was only provided with angiograms. This interventionalist was blinded to outcomes, presentation, or any demographic data. Recanalization time was defined as time from groin access to achieving at least TICI 2B flow within the affected territory. Post-procedural head CT and MRI reports were reviewed for any concern for intracranial hemorrhage, even in the event of likely contrast extravasation. Concerning scans were scored for intracranial hemorrhage using the European Cooperative Acute Stroke Study 1 and 2 grading scales by an independent reviewer (JV). ${ }^{14}$ The modified Rankin Scale 
Figure 1 Schematic of the A Direct Aspiration First Pass Technique (ADAPT) for thrombectomy. A Neuron Max 088 is positioned in the petrous carotid for proximal support. In a triaxial fashion, an 064 ACE reperfusion catheter (Penumbra Inc, Alamade CA) is advanced to the level of the occlusion over a 3 MAX reperfusion catheter (Penumbra Inc, Alamade CA) and a 0.016" Fathom microwire (Boston Scientific, Marlborough MA). The delivery microcatheter and microwire are removed, and the 064 ACE catheter is advanced further to engage the thrombus. The catheter is then connected to aspiration tubing and aspiration is turned on while the catheter is left in place for a few minutes. Lack of blood return in the aspiration tubing confirms clot engagement at the orifice of the aspiration catheter. Typically, the thrombus will be ingested into the catheter and into the canister of the aspiration pump. Brisk blood return through the tubing indicates patency of the catheter lumen and confirms clot aspiration. Angiography with injection of contrast through the reperfusion catheter is then performed to verify recanalization of the occluded vessel.

(mRS) score at 90 days was obtained from the neurology clinic record, which was administered by certified stroke neurologists.

\section{Patient selection}

At our institution, mechanical thrombectomy is the preferred treatment modality for AIS patients who do not qualify for intravenous tissue plasminogen activator (IV tPA), do not improve with IV tPA, or have severe strokes with LVO. Candidacy for intervention is determined by CT perfusion imaging, irrespective of time of onset. ${ }^{15}$

\section{Technique}

ADAPT has been described previously (figure 1). ${ }^{11}$ Briefly, access to the cerebral vasculature is obtained and a large guide catheter (Neuron 088 Max; Penumbra, Oakland, California, USA) is advanced as far distally into the internal carotid artery (ICA) or vertebral artery of interest as safely as possible to anchor it around turns. The largest caliber aspiration catheter that the vessel can accommodate is selected for each case, and the catheter is advanced to the level of the thrombus over a microwire, most commonly a Velocity microcatheter (Penumbra Alameda, California, USA) over a 0.016 inch Fathom wire (Boston Scientific Corp, Naidich, Massachusetts, USA). Aspiration is applied by use of the Penumbra aspiration pump that is part of the Penumbra system. Inability to draw back on aspiration confirms the optimal position of the aspiration catheter abutting the thrombus. At this point, the catheter is slightly advanced to ensure firm engagement with the thrombus. 
For M1 thrombi, the 5 MAX, 5 MAX ACE, and 064 catheters were primarily employed. The aspiration catheter is then slowly withdrawn while maintaining constant aspiration. In smaller caliber or more distal vessels, the technique is employed with either a 4 MAX or 3 MAX reperfusion catheter (Penumbra Alameda). Additional devices such as stent retreivers were used at the discretion of the operator if aspiration failed. All operators selected patients for thrombectomy according to their usual protocol which employed CT perfusion imaging irrespective of time from symptom onset. ${ }^{15} 16$

For statistical analysis, the Student's $\mathrm{t}$ test was used to compare means, and Fischer's exact test for comparing significance of groups of outcomes. A p value of $<0.05$ was accepted as statistically significant.

\section{RESULTS}

\section{Patients}

From December 2012 to June 2015, 191 consecutive patients who suffered an AIS were treated with ADAPT by four operators. Preoperative metrics, procedural details, and outcome data are presented in table 1.

Ninety-one patients were women (47.6\%), and the average age was 67 (range 27-93 years, \pm 14 years). A total of 171 $(89.5 \%)$ ischemic strokes occurred in the anterior circulation and $20(10.5 \%)$ in the posterior circulation. Patients presented with a mean NIHSS score of 15.4 (range $0-36, \pm 7.0$ ), and 71 $(37.2 \%)$ patients received IV tPA. The average time from onset to puncture was $7.8 \mathrm{~h}$ (range $20 \mathrm{~min}$ to $>36 \mathrm{~h}, \pm 6.1 \mathrm{~h}$ ). Twenty-three patients $(12.0 \%)$ presented as wakeup strokes. The average length of stay was 9.3 days (range $0-193, \pm 16.9$ days). Twelve $(6.3 \%)$ patients had tandem lesions noted during treatment. In 10 cases, the occlusion was in the ICA, with tandem distal lesions in ipsilateral branches of the middle cerebral artery. There were no cases of emboli to new territory following aspiration thrombectomy. In two cases of basilar artery

\begin{tabular}{lc} 
Table $1 \quad$ Patient population & \\
\hline Gender $(n=191)(n(\%))$ & $100(52.4)$ \\
Male & $91(47.6)$ \\
Female & $67( \pm 14)$ \\
Age (years) (mean \pm SD) & $15.4( \pm 7)$ \\
NIHSS (mean $\pm S D)$ & \\
Side ( $n=191)(n(\%))$ & $95(49.7)$ \\
Left & $76(39.8)$ \\
Right & $20(10.5)$ \\
Midline & \\
Location ( $n=191)(n(\%))$ & $6(3.1)$ \\
CCA & $34(17.8)$ \\
ICA & $130(68.1)$ \\
MCA & $1(0.5)$ \\
ACA & $1(0.5)$ \\
Vertebral & $19(9.9)$ \\
Basilar & $2.58( \pm 2.07)$ \\
Mean 90 day mRS (mean $\pm S D)$ & \\
90 day mRS ( $n=181)(n(\%))$ & $98(54.1)$ \\
0-2 & $56(30.9)$ \\
$3-5$ & $27(14.9)$ \\
6 & $9.3( \pm 16.9)$ \\
Length of stay (days) (mean $\pm S D)$ & \\
\hline ACA, anterior cerebral artery; CCA, common carotid artery; ICA, internal carotid \\
artery; MCA, middle cerebral artery; mRS, modified Rankin Scale; NIHSS, National \\
Institutes of Health Stroke Scale.
\end{tabular}

occlusion, the patients had additional occlusion of left sided middle cerebral artery branches, one of which was felt to be a chronic thrombus and was not aspirated.

\section{Recanalization}

The average time for recanalization was 37.3 min (range 7-160 min, \pm 29.6 ) across all cases. There were no differences among operators. Independent adjudicated angiographic outcomes of TICI 2B or better recanalization was achieved in $180(94.2 \%)$ patients, TICI 2C or better was achieved in $157(82.2 \%)$ patients, and TICI 3 was achieved in 85 patients (44.5\%). Recanalization was unsuccessful in two cases. In one patient who suffered a basilar occlusion from extensive atherosclerotic plaque, recanalization was not achieved (TICI 0) due to abortion of the procedure secondary to intraprocedural basilar artery rupture. In a second patient, multiple attempts were made to recanalize a completely occluded right ICA, ultimately resulting in partial revascularization.

The ACE 64 catheter was used in seven cases, all of which achieved a TICI score of $2 \mathrm{C}$ or better (100\%). The 5 MAX ACE was used in 101 cases, of whom 78 (77.2\%) achieved TICI 2C or better. The 5 MAX catheter was used in 33 cases, of whom $30(90.2 \%)$ achieved TICI 2C or better. The likelihood of achieving TICI $2 \mathrm{C}$ or better recanalization was not significant for the ACE $64(p=0.35), 5$ MAX ACE $(p=0.087)$, or 5 MAX $(p=0.21)$ catheters.

\section{Complications}

There were four intraprocedural complications. In one case, the patient presented with basiliar artery occlusion due to severe atherosclerotic disease. The case was complicated by tortuous aortic arch and vertebrobasilar artery anatomy that made access difficult to the site of occlusion. Once direct aspiration failed and underlying atherosclerosis was suspected, serial balloon angioplasty was performed along the length of the basilar artery which ultimately resulted in basilar artery rupture. This patient ultimately died. Another case underwent angioplasty and stenting of an occluded left ICA, as well as left M2 and A2 occlusions. During the treatment of the intracranial occlusions, the patient suffered a dissection of the left ICA which was treated with an enterprise stent. This patient suffered no neurological deficits from the dissection, and the 90 day mRS score was 0 . A second flow limiting right ICA dissection was treated with stenting during aspiration of an M1 occlusion. This patient suffered no new neurological symptoms from this lesion, and the 90 day $\mathrm{mRS}$ score was 2 . In another case, the patient presented with left supraclinoid ICA occlusion and underwent thrombectomy and stenting. During the procedure the patient had ST depression, with elevation in his troponin level to 7. Cardiology was consulted, who recommended medical management. The patient was discharged to a long term acute care facility, with a 90 day mRS score of 5 . Thirteen $(6.8 \%)$ patients suffered clinically significant postprocedural $\mathrm{PH} 2$ hemorrhages. One $(0.57 \%)$ patient had a retroperitoneal hematoma. Seven (3.7\%) patients suffered postprocedural gastrointestinal bleeds.

\section{Functional outcomes}

The 90 day mRS score was available for 181 patients $(94.8 \%)$, and 10 patients were lost to follow-up. Mean follow-up mRS was 2.58 (range $0-6, \pm 2.07$ ). A total of $98(54.1 \%)$ patients had an mRS score of $0-2$ at 90 days, and 27 (14.9\%) patients had expired (mRS of 6 ) by the 90 day follow-up. Of the 171 anterior circulation ischemic strokes, 90 day mRS was available 
Table 2 Direct aspiration alone versus direct aspiration plus additional devices

\begin{tabular}{|c|c|c|c|}
\hline & $\begin{array}{l}\text { Direct } \\
\text { aspiration only } \\
(n=145)\end{array}$ & $\begin{array}{l}\text { Direct aspiration } \\
\text { and adjunct } \\
(n=43)\end{array}$ & p Value \\
\hline Age (years) $($ mean $\pm S D)$ & $65( \pm 14.2)$ & $72.4( \pm 13.2)$ & 0.0019 \\
\hline $\begin{array}{l}\text { Time from onset to } \\
\text { groin puncture (min) } \\
\text { (mean } \pm S D)\end{array}$ & $483.9( \pm 365.1)$ & $407.8( \pm 288.4)$ & 0.23 \\
\hline NIHSS score $(m e a n \pm S D)$ & $15.27( \pm 7)$ & $15.69( \pm 6.6)$ & 0.72 \\
\hline $\begin{array}{l}\text { Time to recanalization } \\
\text { (min) }(\text { mean } \pm S D)\end{array}$ & $30.1( \pm 29.6)$ & $61.4( \pm 35.9)$ & 0.00000201 \\
\hline \multicolumn{4}{|l|}{ Final TICI (n (\%)) } \\
\hline 0 & $0(0.0)$ & $0(0.0)$ & \\
\hline 1 & $0(0.0)$ & $1(2.2)$ & \\
\hline $2 A$ & $4(2.7)$ & $6(13.3)$ & \\
\hline $2 B$ & $18(12.3)$ & $5(11.1)$ & \\
\hline $2 C$ & $58(39.7)$ & $14(31.1)$ & \\
\hline 3 & $66(45.2)$ & $19(42.2)$ & \\
\hline mRS (n (\%)) & $n=137$ & $\mathrm{n}=44$ & \\
\hline $0-2$ & $79(57.7)$ & $19(43.2)$ & 0.12 \\
\hline $3-5$ & $39(28.5)$ & 17 (38.6) & \\
\hline 6 & 19 (13.9) & $8(18.2)$ & 0.47 \\
\hline
\end{tabular}

in $161(94.2 \%) ; 89(49.2)$ had an mRS of $0-2$, and there were 25 mortalities (14.6\%).

\section{Direct aspiration alone and direct aspiration with adjunct devices}

ADAPT was successful in achieving final recanalization in 145 of cases, and 43 cases required the additional use of a stent retriever (table 2). Eight direct aspiration only cases had lesions in more than one vessel, compared with four in the direct aspiration with adjunctive devices. In two cases, recanalization was not achieved. For direct aspiration only cases, the average age was significantly different from that in which an additional device was employed to achieve recanalization (64.95 years vs 72.38 years; $p=0.0019)$. Mean time to recanalization was significantly faster for direct aspiration only cases, with revascularization achieved in $30.12 \mathrm{~min}$ (range 7-150 min, \pm 29.56 ) compared with $61.40 \mathrm{~min}$ (range 8-160 min, \pm 35.85 ) $(\mathrm{p}=0.00000201)$. There were no significant differences in likelihood of achieving a 90 day mRS of $0-2 \quad(57.7 \%$ for direct aspiration only vs $43.2 \% ; \mathrm{p}=0.12$ ) or mortality rates among the two groups $(13.9 \%$ for direct aspiration only vs $18.2 \%$; $\mathrm{p}=0.47)$.

\section{DISCUSSION}

Direct aspiration alone has been previously described in the literature but usually as a last resort after more conventional techniques have failed. ${ }^{17-20}$ Newer, large bore, flexible catheters not only allow direct aspiration as the primary mechanism for thrombectomy but also provide a platform for incorporating secondary devices such as stent retrievers if direct aspiration fails. These catheters are able to generate an aspiration rate that allows for removal of thrombus without the use of a separator or retriever. ${ }^{21}$ The use of direct aspiration first has been successfully reported in other series, and has been shown to be more cost effective. ${ }^{22}$ Despite these promising results, the use of direct aspiration with large bore catheters has not yet been studied in large trials.

We have previously reported our experience as part of a multicenter collaborative effort using ADAPT as a rapid method for quickly achieving good revascularization using large bore aspiration catheters. ${ }^{10} 11$ This current series are consecutive patients and did not adhere to the strict patient selection of recent trials (average time to recanalization $468.3 \mathrm{~min}$ ). We found that $94.2 \%$ of all cases were successfully revascularized to TICI $2 \mathrm{~B}$ or better, with 145 cases successfully treated with direct aspiration alone; 43 cases required the use of adjunct devices such as stent retrievers. The original TICI score is limited in that it does not differentiate between perfusion of $51 \%$ of the ischemic territory (TICI 2B) and complete perfusion (TICI 3). To address this issue we have started using the term TICI $2 \mathrm{C}$ for situations when the angiogram is nearly normal (a few distal branches demonstrate slow flow or a tiny distal embolus). When utilizing the revised TICI scale, a grade of TICI $2 \mathrm{C}$ or better was achieved in $84.9 \%$ of cases of direct aspiration alone and in $73.3 \%$ of cases where adjunctive devices were required, and cases where an additional device was used required a longer mean time to recanalization (21.3 additional minutes). ${ }^{13}$

Twelve patients had tandem occlusions identified during initial treatment. In 10 cases the tandem lesion was ipsilateral and downstream of the initial thrombus. It is difficult to establish whether these lesions were present before aspiration or as a result of treatment, since ADAPT does not require crossing the target thrombus prior to treating, as is the case with stentrievers. In the two cases with basilar artery occlusions with additional lesions in the anterior circulation, these were present on initial CT angiography and as such do not represent new territory emboli.

The longer recanalization times in the direct aspiration and adjunctive devices group implies a subgroup of patients with more complex or difficult thromboembolic pathology and/or underlying atherosclerotic disease, evidenced by the overall lower quality of thrombectomy and longer time to achieve recanalization. Importantly, despite the significant age difference and longer time to revascularization, there was no significant difference in outcomes between the two groups. This suggests that if direct aspiration alone is unsuccessful in achieving recanalization, using an additional device can yield good angiographic and clinical outcomes in patients with challenging pathology.

The rates of good outcome in this series were comparable with those in the recent trials, with $54.14 \%$ patients obtaining an mRS score of $0-2$ at 90 days. Angiographic outcomes were also similar, with $94.2 \%$ of patients achieving TICI 2 B. These findings are very encouraging considering our patient selection represents a liberal real world experience rather than a population selected with stringent trial based inclusion and exclusion criteria. Early recanalization has been associated with better patient outcomes, and trials such as Solitaire With the Intention For Thrombectomy as Primary Endovascular Treatment (SWIFT PRIME), Extending the Time for Thrombolysis in Emergency Neurological Deficits-Intra-Arterial (EXTEND-IA), Multicenter Randomized Clinical trial of Endovascular treatment for Acute ischemic stroke in the Netherlands (MR CLEAN), Endovascular Revascularization With Solitaire Device Versus Best Medical Therapy in Anterior Circulation Stroke Within 8 Hours (REVASCAT), and Endovascular Treatment for Small Core and Proximal Occlusion Ischemic Stroke (ESCAPE) have focused on rapid patient selection and reperfusion. ${ }^{1-6} 23-25$ In ESCAPE and EXTEND-IA, the median times from onset of symptoms to reperfusion were 241 and $248 \mathrm{~min}$, respectively, and the median times from groin puncture to revascularization were 56 and 
43 min. The ADAPT recanalization time from groin puncture of $37.3 \mathrm{~min}$ for all cases was slightly faster than in REVASCAT or EXTEND-IA, and slightly longer than in the ESCAPE trial. While SWIFT PRIME reported a time from groin puncture to first stent deployment of $28 \mathrm{~min}$, the authors did not report a time from groin puncture to TICI $2 \mathrm{~B}$ or better, so a comparison between recanalization times is difficult. The results of our series provide further evidence that the speed of revascularization is more important than time to presentation when an imaging based selection process is used, and supports the findings from the large thrombectomy trials, in which average time to recanalization was at most $43 \mathrm{~min}$, resulting in significantly more patients achieving an mRS of 0-2 compared with controls. Mortality in our series was comparable. In addition, this series included many patients with tandem occlusions and posterior circulation strokes which are known to have worse outcomes. ${ }^{23-25}$ This suggests that posterior circulation lesions and tandem occlusions can also be treated successfully and should be considered for intervention.

This case series is limited by its retrospective nature, as well as a lack of a control group. Revascularization was assessed by a blinded neurointerventionalist to eliminate bias but at follow-up the clinicians assessing the 90 day mRS scores were not blinded to the techniques and devices used. Ten patients were lost to follow-up, representing a 5.2\% loss. Additionally, our institution is a high volume stroke center with extensive stroke resources, including a telehealth network and experienced neurointerventionalists. The results of these studies may not be generalizable to centers without such resources.

\section{CONCLUSIONS}

ADAPT is an efficient method to achieve good clinical and angiographic outcomes, with $82.2 \%$ of patients achieving TICI 2C or better recanalization and 90 day mRS scores of $0-2$ in $54.4 \%$. These results are comparable with recent randomized trials that demonstrate the benefit of intra-arterial thrombectomy. Further, this series demonstrates good clinical outcomes in this unselected patient cohort, including many beyond the $6 \mathrm{~h}$ cut-off used in these recent studies. ADAPT as a firstline strategy for achieving revascularization in the setting of acute LVO should be further studied in a randomized trial.

Contributors Each author should receive authorship credit based on material contribution to this article, their revision of this article, and their final approval of this article for submission to this journal. Alyssa Pierce assisted with the editing and revision of this manuscript.

Competing interests AS: Penumbra—consulting, honorarium, speaker bureau; Pulsar Vascular-consulting, honorarium, speaker bureau; Microvention-consulting, honorarium, speaker bureau, research; Stryker—consulting, honorarium, speaker bureau. AT, RT, and IC: Codman — consulting, honorarium, speaker bureau, research funding; Covidien—consulting, honorarium, speaker bureau; Penumbra—consulting, honorarium, speaker bureau, research grants; Microvention-consulting, honorarium, speaker bureau, research grants; Blockade — stock, consulting, honorarium, speaker bureau; Pulsar Vascular-stock, consulting, honorarium, speaker bureau, research; Medtronic — consulting, honorarium, speaker bureau.

Ethics approval The study was approved by the institutional review board of Medical University of South Carolina.

Provenance and peer review Not commissioned; externally peer reviewed.

\section{REFERENCES}

1 Rha JH, Saver JL. The impact of recanalization on ischemic stroke outcome: a meta-analysis. Stroke 2007;38:967-73.

2 Goyal M, Demchuk AM, Menon BK, et al. Randomized assessment of rapid endovascular treatment of ischemic stroke. N Engl J Med 2015;372:1019-30.

3 Saver JL, Goyal M, Bonafe A, et al. Stent-retriever thrombectomy after intravenous t-PA vs. t-PA alone in stroke. N Engl I Med 2015;372:2285-95.

4 Campbell BC, Mitchell PJ, Kleinig TJ, et al. Endovascular therapy for ischemic stroke with perfusion-imaging selection. N Engl J Med 2015;372:1009-18.

5 Berkhemer OA, Fransen PS, Beumer $\mathrm{D}$, et al. A randomized trial of intraarterial treatment for acute ischemic stroke. $N$ Engl J Med 2015;372:11-20.

6 Jovin TG, Chamorro A, Cobo E, et al. Thrombectomy within 8 hours after symptom onset in ischemic stroke. N Engl J Med 2015;372:2296-306.

7 Mocco J, Fiorella D, Fargen KM, et al. Endovascular therapy for acute ischemic stroke is indicated and evidence based: a position statement. J Neurointerv Surg 2015:7:79-81.

8 Nogueira RG, Lutsep HL, Gupta R, et al. Trevo versus Merci retrievers for thrombectomy revascularisation of large vessel occlusions in acute ischaemic stroke (TREVO 2): a randomised trial. Lancet 2012;380:1231-40.

9 Saver JL, Jahan R, Levy El, et al. Solitaire flow restoration device versus the Merci retriever in patients with acute ischaemic stroke (SWIFT): a randomised, parallel-group, non-inferiority trial. Lancet 2012;380:1241-9.

10 Turk AS, Frei D, Fiorella D, et al. ADAPT FAST study: a direct aspiration first pass technique for acute stroke thrombectomy. I Neurointerv Surg 2014;6:260-4.

11 Turk AS, Spiotta A, Frei D, et al. Initial clinical experience with the ADAPT technique: a direct aspiration first pass technique for stroke thrombectomy. J Neurointerv Surg 2014;6:231-7.

12 Higashida RT, Furlan AJ, Roberts $H$, et al. Trial design and reporting standards for intra-arterial cerebral thrombolysis for acute ischemic stroke. Stroke 2003;34: e109-37.

13 Goyal $\mathrm{M}$, Fargen $\mathrm{KM}$, Turk $\mathrm{AS}$, et al. $2 \mathrm{C}$ or not $2 \mathrm{C}$ : defining an improved revascularization grading scale and the need for standardization of angiography outcomes in stroke trials. J Neurointerv Surg 2014;6:83-6.

14 Larrue V, von Kummer RR, Müller A, et al. Risk factors for severe hemorrhagic transformation in ischemic stroke patients treated with recombinant tissue plasminogen activator: a secondary analysis of the European-Australasian Acute Stroke Study (ECASS II). Stroke 2001;32:438-41.

15 Turk AS, Magarick JA, Frei D, et al. CT perfusion-guided patient selection for endovascular recanalization in acute ischemic stroke: a multicenter study. J Neurointerv Surg 2013;5:523-7.

16 Turk AS, Nyberg EM, Chaudry MI, et al. Utilization of CT perfusion patient selection for mechanical thrombectomy irrespective of time: a comparison of functional outcomes and complications. J Neurointerv Surg 2013;5:518-22.

17 Iwata T, Mori T, Tajiri H. [Successful removal of clots from embolic carotid occlusion by aspiration with a syringe--case report]. Brain Nerve 2008;60:1067-72.

18 Jankowitz B, Aghaebrahim A, Zirra A, et al. Manual aspiration thrombectomy: adjunctive endovascular recanalization technique in acute stroke interventions. Stroke 2012;43:1408-11.

19 Xu GF, Suh DC, Choi CG, et al. Aspiration thrombectomy of acute complete carotid bulb occlusion. J Vasc Interv Radio/2005;16:539-42.

20 Jankowitz BT, Aleu A, Lin R, et al. Endovascular treatment of basilar artery occlusion by manual aspiration thrombectomy. I Neurointerv Surg 2010;2:110-14.

21 Hu YC, Stiefel MF. Force and aspiration analysis of the ADAPT technique in acute ischemic stroke treatment. J Neurointerv Surg 2016;8:244-6.

22 Comai A, Haglmuller T, Ferro F, et al. Sequential endovascular thrombectomy approach (SETA) to acute ischemic stroke: preliminary single-centre results and cost analysis. Radiol Med 2015;120:655-61.

23 Hassan AE, Chaudhry SA, Miley JT, et al. Microcatheter to recanalization (procedure time) predicts outcomes in endovascular treatment in patients with acute ischemic stroke: when do we stop? AJNR 2013;34:354-9.

24 Spiotta AM, Vargas J, Turner $\mathrm{R}$, et al. The golden hour of stroke intervention: effect of thrombectomy procedural time in acute ischemic stroke on outcome. J Neurointerv Surg 2014;6:511-16.

25 Sun $\mathrm{CH}$, Nogueira RG, Glenn BA, et al. "Picture to puncture": a novel time metric to enhance outcomes in patients transferred for endovascular reperfusion in acute ischemic stroke. Circulation 2013;127:1139-48. 